Full Length Article

\title{
A facile single injection Hydrothermal method for the synthesis of thiol capped CdTe Quantum dots as light harvesters
}

\author{
B. Jai Kumar, D. Sumanth Kumar, H.M. Mahesh* \\ Department of Electronic Science, Thin film solar cell Lab, Bangalore University, Jnanabharathi campus, Bangalore 560056, India
}

\section{A R T I C L E I N F O}

\section{Article history:}

Received 29 October 2015

Received in revised form

13 May 2016

Accepted 3 June 2016

Available online 9 June 2016

\section{Keywords:}

MPA capped CdTe Quantum dots

Single injection Hydrothermal method

Quantum confinement effect

Optical property

Aqueous synthesis

\begin{abstract}
A B S T R A C T
A facile, Single Injection Hydrothermal (SIH) method has been developed to synthesize high quality 3Mercaptopropionic Acid (MPA) stabilized aqueous CdTe QDs, entirely in ambient environment. The synthesis protocol eliminates the use of inert atmosphere for reducing elemental Tellurium powder to Te precursor avoiding the oxidation of Te powder. The XRD result revealed that the synthesized QDs are in cubic zincblende type crystalline structure, without signature of Te oxidation. FTIR spectra have confirmed the attachment of short chained organic compound MPA to the surface of QDs by covalent bond. The Quantum confinement effect was clearly evident by shift in Longitudinal Optic (LO) peak of Raman spectra and absorption peak wavelength with respect to bulk CdTe materials. The optical direct band gap energy of CdTe QDs is between $3.63 \mathrm{eV}$ to $1.96 \mathrm{eV}$ and QDs size below $6 \mathrm{~nm}$, confirm the QDs are well under strong Quantum confinement regime. Also, photoluminescence spectra depict a stable and high luminescence emission from green to dark red color. All these results corroborate that the synthesis of CdTe QDs procedure is very advantageous and present a simple, economical and easily up scalable method for large scale production.
\end{abstract}

(c) 2016 Elsevier B.V. All rights reserved.

\section{Introduction}

Semiconductor Quantum dots, zero dimension Semiconductor Nanocrystals are the materials, in which motion of electron and holes are confined in all three spatial dimensions. This phenomenon leads to renowned quantum size effect or quantum confinement effect by which nanocrystals owes its name Quantum Dots (QDs) [1-3]. QDs whose size is less than Bohr exciton radius of the material exhibits interesting characteristic properties than its bulk counterpart, as continuous density of states in bulk collapse into discrete electronic state leading to size tunable band gap in QDs. This size tunable band gap and large surface to volume ratio of QDs gives rise to unique and remarkable properties which were not observed in bulk semiconductor materials [1-3].

In recent years, the quest for alternative solar energy harvesting material has become intense. In this context, the QDs are considered as future prospective material due to their distinctive properties like Multiple Exciton Generation (MEG), larger extinction coefficient and specifically tailored band gap [1-3]. It is predicted that these semiconductor nanocrystals based assemblies can achieve the energy

\footnotetext{
Abbreviations: SIH, Single injection Hydrothermal; CdTe, Cadmium Telluride; QDs, Quantum dots; MEG, Multiple exciton generation; NaHTe, Sodium Telluride; $\mathrm{Cd}\left(\mathrm{NO}_{3}\right)_{2}$, Cadmium nitrate; Te, Tellurium; $\mathrm{NaBH}_{4}$, Sodium Borohydride; MPA, 3Mercaptopropionic acid

* Corresponding author.

E-mail address: hm_mahesh@rediffmail.com (H.M. Mahesh).
}

conversion efficiency much beyond Shockley Queisser limit of 31\% for traditional Si based solar cell [4]. In addition, the same QDs also find application in biomedical labeling, imaging, sensing and diagnostics by utilizing their fluorescence properties [5,6]. Among various semiconductor QDs known till date, Cadmium telluride (CdTe) QDs is one of most significant semiconductor nanocrystals which are largely used in solar photovoltaic cell, opto-electronic devices and in vivo biomedical detection fluorescent tags in biology [5-8].

Colloidal synthesis is one of the important and extensively studied methods for synthesizing QDs, with advantages of solution processing and easy tuneability of size for desired band gap. This can be further classified as aqueous and non-aqueous solvent synthesis routes. Aqueous route is advantages than non-aqueous (organometallic) as it can be performed in low temperature, less toxic and bio-compatible [5,6,9-13]. The aqueous CdTe synthesis mainly comprises two steps, first preparation of Te precursor and followed by growth of CdTe QDs. Te Precursor is prepared by reducing elemental Te in $\mathrm{N}_{2}$ /inert atmosphere to prevent oxidation of Te [5,6], which increases the cost of reaction. To prevent this, researchers are trying to synthesis CdTe QDs using precursors like $\mathrm{Al}_{2} \mathrm{Te}_{3}$ [13,14], Te (as electrode) [9,10], $\mathrm{Na}_{2} \mathrm{TeO}_{3}[11,12]$, etc. But, $\mathrm{Al}_{2} \mathrm{Te}_{3}$ decompose chemically to produce highly unstable gas $\mathrm{H}_{2}$ Te which makes the reaction tedious. Using Te electrode in electrochemical synthesis is clean but, it's hard to obtain complete size range. $\mathrm{Na}_{2} \mathrm{TeO}_{3}$ has been used recently but, it is expensive than elemental Te and the availability is less. Hence to overcome these practical difficulties, a facile method 
has been developed in which Te powder is reduced by $\mathrm{NaBH}_{4}$ to form NaHTe without oxidation of Te, resulting in synthesis of CdTe QDs completely in ambient atmosphere. Here, we are reporting the synthesis of CdTe QDs by SIH method in ambient environment using MPA as capping agent. This approach can be easily extended to largescale aqueous synthesis as this procedure is quicker, most efficient and low cost. Literatures on synthesis of CdTe QDs in ambient atmosphere are sparse and especially this method of synthesis report is first of this kind.

\section{Experimental details}

\subsection{Materials}

High purity Cadmium nitrate $\left(\mathrm{Cd}\left(\mathrm{NO}_{3}\right)_{2}, 99 \%\right.$, Aldrich), Tellurium powder (Te, - 200 mesh, 99.8\%, Aldrich), Sodium Borohydride $\left(\mathrm{NaBH}_{4}, 98 \%\right.$, Aldrich), MPA $\left(\mathrm{C}_{3} \mathrm{H}_{6} \mathrm{O}_{2} \mathrm{~S}, 99 \%\right.$, Spectrochem), Ammonium hydroxide $\left(\mathrm{NH}_{4} \mathrm{OH}\right.$, Merck) and Sodium hydroxide $(\mathrm{NaOH}$, Spectrochem), solvent - distilled water, were used for the synthesis of QDs.

\subsection{Synthesis of MPA capped CdTe QDs}

CdTe QDs were synthesized using SIH method, which can be explained in 3 stages. During the first stage MPA capped Cadmium (Cd) precursor was prepared by adding $5 \mathrm{ml}$ of $0.04 \mathrm{M}$ MPA in to $10 \mathrm{ml}$ distilled water and stirred for $5 \mathrm{~min}$, followed by addition of Cadmium nitrate $0.04 \mathrm{M} 5 \mathrm{ml}$ solution and stirred for $10 \mathrm{~min}$. The $\mathrm{pH}$ of solution was adjusted to 9 by drop wise addition of $\mathrm{NaOH}$ or $\mathrm{NH}_{4} \mathrm{OH}$ of $1 \mathrm{M}$ solution with rigorous stirring for $30 \mathrm{~min}$, and was continued till Te precursor was ready. In the second stage, Te source was prepared by adding $0.2 \mathrm{M} 5 \mathrm{ml} \mathrm{NaBH}_{4}$ to $5 \mathrm{ml}$ distilled water and then $\mathrm{NaBH}_{4}$ was completely dissolved, followed by addition of equivalent weight quantity of $(0.08 \mathrm{M}$ for $5 \mathrm{ml})$ Tellurium powder directly to above $\mathrm{NaBH}_{4}$ solution. The flask was tightly closed, shacked thoroughly once and kept in preheated water bath at $80^{\circ} \mathrm{C}$. Few small holes were poked onto flask cap to allow the reaction gas to escape. Within a minute, dark gray solution turn to clear solution with liberation of $\mathrm{H}_{2}$ gas, this tiny gas bubbles intensify as reaction proceeds. Gradually, clear solution changes to dark pinkish-violet color; indicating that Te precursor is ready. At this stage, $2 \mathrm{ml} \mathrm{Te}$ precursor (from $10 \mathrm{ml}$ ) was injected to Cd solution under stirring. The Cd solution turns to dark golden color indicating nucleation of CdTe. This solution was stirred for $10 \mathrm{~min}$ at room temperature and then on preheated hot plate at $90{ }^{\circ} \mathrm{C}$. After $10 \mathrm{~min}$ of reflux, green color QDs appeared and as reflux time increases yellow, orange, red and finally brick red colored QDs appears, when excited by $365 \mathrm{~nm}$ UV light source. The CdTe QDs aliquots were collected and quenched at regular interval and further processed before characterization.

\subsection{Post synthesis steps before characterization}

Synthesized QDs were precipitated using acetone to remove un-reacted and excess bi-products by maintaining the CdTe solution to acetone at the optimized ratio $1: 2$ and centrifuged at $3000 \mathrm{rpm}$ to get dots. The solid remain of QDs was re-dispersed in water and the above process was repeated twice. Further QDs were washed with ethanol trice as above and, finally CdTe QDs powder was obtained by vacuum drying.

\subsection{Instruments used for characterizations}

CdTe QDs were characterized by Rigaku smartlab XRD system for crystal structure, in $2 \theta$ range $20-70^{\circ}$ with step size of $0.002^{\circ}$. Raman spectra was obtained by LabRAM HR spectrometer excited by $514 \mathrm{~nm}$ wave length laser with spectral resolution of $1 \mathrm{~cm}^{-1}$, used to study Quantum confinement. MPA capping was verified using Bruker-Alpha Fourier Transform Infrared Spectroscopy (FTIR) solid samples in transmittance mode, in range 4000$400 \mathrm{~cm}^{-1}$. Optical properties were measured using Ocean optics USB 4000 UV-Vis-NIR spectrophotometer and Photoluminescence (PL) by LabRAM HR spectrometer.

\section{Result and discussion}

The synthesis of MPA stabilized CdTe QDs was accomplished through 3 stages which has been explained in brief in the previous section, in first stage MPA and $\mathrm{Cd}$ solution are added and the $\mathrm{pH}$ is balanced to 9 . The detailed reaction is as follow Cadmium nitrate reacts with $\mathrm{NaOH} / \mathrm{NH}_{4} \mathrm{OH}$ resulting in a white precipitate of $\mathrm{Cd}$ $(\mathrm{OH})_{2}$ capped with MPA ( $\left.\mathrm{pH} \sim 5\right)$.

$$
\begin{aligned}
& \mathrm{Cd}\left(\mathrm{NO}_{3}\right)_{2}+2 \mathrm{NH}_{4} \mathrm{OH} \quad(\text { or } \quad 2 \mathrm{NaOH}) \rightarrow \mathrm{Cd}(\mathrm{OH})_{2}+2 \mathrm{NH}_{4} \mathrm{NO}_{3} \\
& \left.2 \mathrm{NaNO}_{3}\right)
\end{aligned}
$$

when $\mathrm{NH}_{4} \mathrm{OH}$ is further added, $\mathrm{Cd}(\mathrm{OH})_{2}$ gets dissolved forming Cadmium cations $\left(\mathrm{Cd}^{2+}\right)$ encapsulated with MPA and hydroxyl ion $(\mathrm{OH}-)$ which result in clear solution $(\mathrm{pH} \sim 7)$.

$\mathrm{Cd}(\mathrm{OH})_{2}+4 \mathrm{NH}_{4} \mathrm{OH} \rightarrow \mathrm{Cd}^{2+}+$ (other bi-products)

with small addition of $\mathrm{NaOH} \mathrm{pH}$ reaches 9 . If $\mathrm{pH}>12$, white precipitate is formed and is reversible but, process of reversibility is tedious. The $\mathrm{pH}$ of solution should be maintained between $\mathrm{pH} 8$ 12 wherein, MPA capped Cadmium precursor will be clear and results in good chemical yield. The capping agent MPA will surround the $\mathrm{Cd}$ cation with bonding between $\mathrm{Cd}$ cation and thiol group of MPA. However, if $\mathrm{pH}$ is greater than 12, sulfur $(\mathrm{S})$ in thiol group may detach and act as $S$ precursor and lead to formation of CdS QDs. Thus, pH plays a vital role in this reaction.

In 2nd Stage Te source was prepared, to start with $\mathrm{NaBH}_{4}$ reduces Te to Sodium Telluride (NaHTe) liberating $\mathrm{H}_{2}$ and NaHTe in aqueous medium release $\mathrm{Te}$ anion $\left(\mathrm{Te}^{2-}\right)$.

$4 \mathrm{NaBH}_{4}+2 \mathrm{Te}+4 \mathrm{H}_{2} \mathrm{O} \rightarrow 2 \mathrm{NaHTe}+11 \mathrm{H}_{2} \uparrow+2 \mathrm{NaBO}_{2}$

In aqueous condition,

$4 \mathrm{BH}^{4-}+2 \mathrm{Te} \rightarrow 2 \mathrm{Te}^{2-}+11 \mathrm{H}_{2} \uparrow+2 \mathrm{BO}^{2-}$

The optimized $\mathrm{NaBH}_{4}$ to Te ratio is 2.5:1. Excess of $\mathrm{NaBH}_{4}$ increases rate of reaction as $\mathrm{Te}$ is reduced faster and conversely, equal or lesser $\mathrm{NaBH}_{4}$ slowdowns the reaction. Te precursor can not be stored in air ambient as it gets oxidized forming black precipitate, but could be stored in inert atmosphere. In this study, interference of oxygen was avoided by covering reaction flask air tight leaving only few vent holes. The reason is to replace oxygen by hydrogen which is liberated in situ as a result of reaction is depicted in Fig. 1. This is a vital step which prevents the use of inert atmosphere or reaction under vacuum. The complete setup was undisturbed until completion of reaction and NaHTe injection. The whole reaction completes in 10 min with 2 min time window to inject mature Te precursor; after which Te precursor starts oxidizing. This window could be extended by reducing water bath temperature below $80^{\circ} \mathrm{C}$ or increasing $\mathrm{NaBH}_{4}$ concentration. In this work, both $\mathrm{Cd}$ and $\mathrm{Te}$ precursor were freshly prepared and used, in complete ambient environment.

In stage $3 \mathrm{CdTe}$ QDs size was controlled in this step. $\mathrm{Cd}^{2+}$ ion starts to bond with $\mathrm{Te}^{2-}$ forming CdTe precursor, with visual indication of CdTe nucleation with changing color, from transparent to golden color. When additional energy (heat) is applied, the reaction boosts up and QDs sizes grow. Thus by monitoring temperature and time of reaction, complete size ranged QDs were collected. The high $\mathrm{Cd}$ to $\mathrm{Te}$ ratio gives advantage in CdTe QDs formation. Also high Cd concentration on QDs surface further helps in covalent bonding 

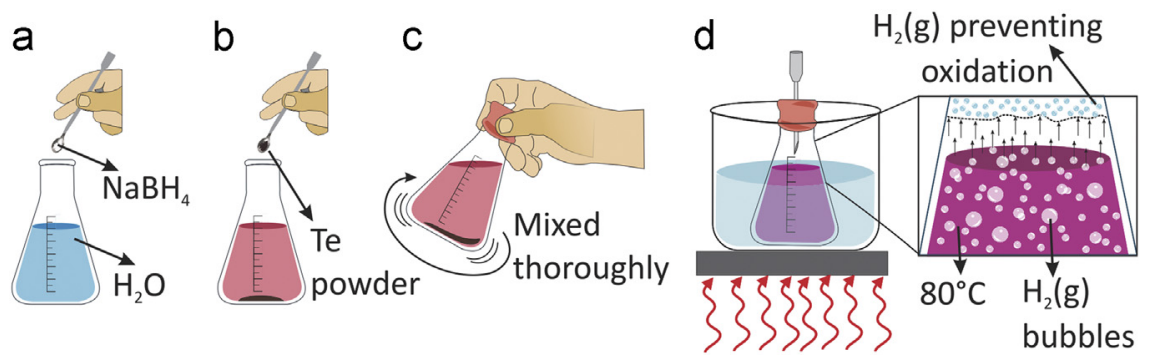

Fig. 1. Schematic diagram of preparing NaHTe by the reduction of Te using $\mathrm{NaBH}_{4}$; preventing oxidation of Te.

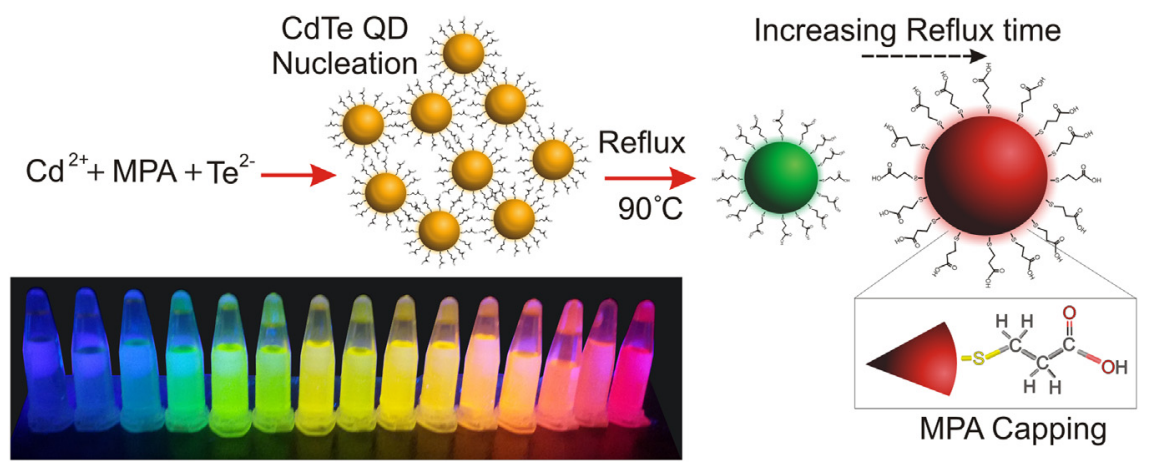

Fig. 2. Graphical illustration of CdTe QDs synthesis process.

between Cd and thiol group of MPA and protect QDs from oxidation. The final reaction (Fig. 2) between $\mathrm{Cd}^{2+}$ and $\mathrm{Te}^{2-}$ is

$\mathrm{Cd}^{2+}-\mathrm{SR}+\mathrm{Te}^{2-} \rightarrow \mathrm{CdTe}-\mathrm{SR}$

\section{n CdTe-MPA $\rightarrow($ CdTe-MPA $)$ n}

Growth of CdTe QDs can be explained by applying classical nucleation and growth model, which could be divided into 3 stages. In the first stage of formation of $\mathrm{Cd}$ and Te precursor, $\mathrm{Cd}\left(\mathrm{NO}_{3}\right)_{2}$ and Te powder decomposes and reduced respectively to form $\mathrm{Cd}^{2+}$ and $\mathrm{Te}^{2-}$ monomers. When these monomers are mixed, it results in fast nucleation stage also called CdTe monomer preparation stage. As Cd and Te concentration reduces Ostwald ripening (OR) stage starts, where small QDs crystals dissolve and then redeposit on the surface of large QDs facilitating growth of larger sized Quantum dots crystals, the driving force of this phase of reaction is, higher surface energy of the small sized QDs, implying high total Gibbs energy, leading to apparently greater solubility than larger sized QDs. As this process continues more stable crystal are formed with lowered total Gibbs energy. Therefore the larger sized QDs are more stable in solution then the smaller ones. Thus, it further increases the reaction time to get large sized QDs.

\section{1. $X R D$}

The XRD pattern of different sized CdTe sample synthesized at different time is shown in Fig. 3. The obtained XRD pattern agrees with JCPDS card number $75-2086$ of CdTe and $2 \theta$ peaks at $24^{\circ}, 39^{\circ}$ and $46.9^{\circ}$ are assigned to planes (111), (200) and (311) respectively. All these confirm synthesized CdTe is in cubic zincblende structure and lattice is Face Centered system. Intensity of XRD peak indicates synthesized CdTe QDs are crystalline and, broad diffraction peaks indicate very small sized crystals. Peak intensity at $2 \theta=24^{\circ}$ show, preferred crystallographic orientation is along 111 plane. The XRD data inter planer spacing (d) between atoms is calculated using Bragg's Law [15] 2d $\sin \theta=n \lambda$; here $n=1$ and $\lambda=1.5418 \AA$ wavelength of Cuk $\alpha$. From d and hkl values, lattice spacing ' $a$ ' is calculated. ' $a$ ' for cubic structure is given by $a^{2}=\left(h^{2}+k^{2}+l^{2}\right) d^{2}$.

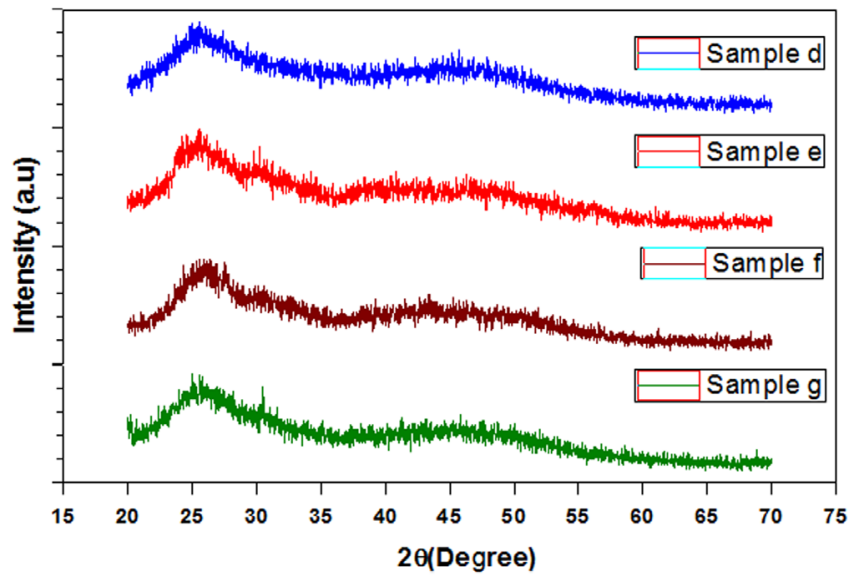

Fig. 3. X- ray powder diffraction patterns of various sized CdTe QDs.

Calculated $a=\sim 6.414 \AA$, which is constant for all $d$ (tabulated in Table 1 ), and agrees with standard value of ' $a$ ' in JCPDS card number is 75-2086 equal to $6.410 \AA$. Also the XRD pattern of CdTe QDs synthesized at different trial shows similar pattern, confirming the synthesis protocol is repeatable with no trace of oxidation of Te.

\subsection{Raman spectra}

Raman spectra of CdTe QDs powder sample is shown in Fig. 4. Here, Quantum confinement is evident by broadening and red shifting of LO.peak. For the current CdTe QDs LO phonon peak is at $162.88 \mathrm{~cm}^{-1}$, which is red shifted by $5.12 \mathrm{~cm}^{-1}$ when compared with bulk CdTe [16]. This pronounced red shift and broadening of Raman spectra should be probably assigned to quantum confinement of CdTe nanocrystals and their uniform size distribution respectively.

\subsection{FTIR analysis}

Successful capping of MPA to the surface of CdTe QDs is confirmed by FTIR analysis (Fig. 5). In MPA capped CdTe QDs, the 
Table 1

Calculated values of $d$ and a along with hkl index of MPA capped CdTe QDs.

\begin{tabular}{llrl}
\hline Peak Position $(2 \theta)$ & $d(\AA)$ & hkl & $a(\AA)$ \\
\hline 24.029 & 3.7034 & 111 & 6.414 \\
39.731 & 2.2686 & 220 & 6.416 \\
46.979 & 1.9341 & 311 & 6.414 \\
57.465 & 1.6036 & 400 & 6.414 \\
63.181 & 1.4716 & 331 & 6.414 \\
\hline
\end{tabular}

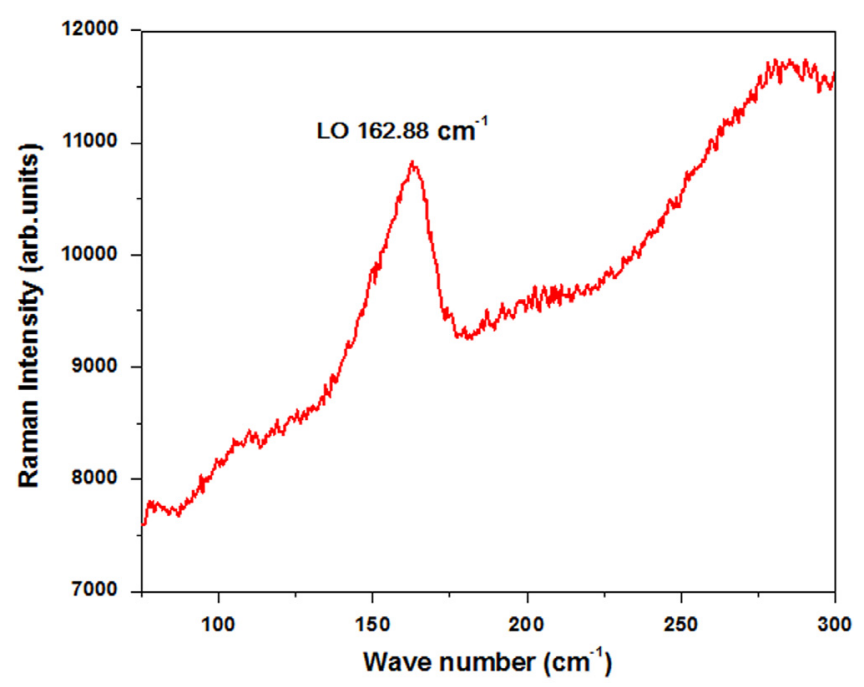

Fig. 4. Raman spectrum of CdTe QDs.

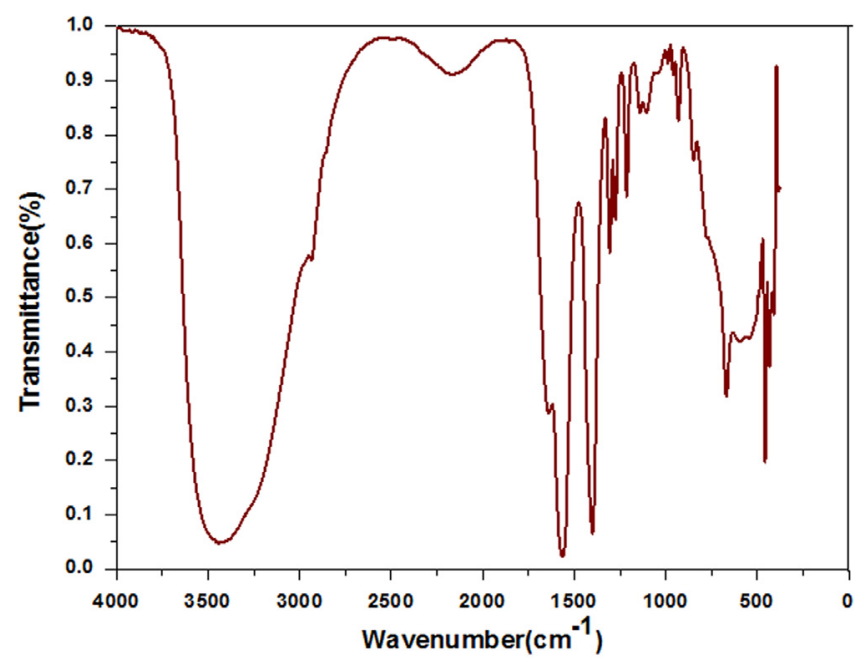

Fig. 5. FTIR spectrum of CdTe QDs.

distinct S-H bond vibration peak of MPA at $2574 \mathrm{~cm}^{-1}$ disappears, confirming the thiol group is bonded covalently to CdTe QDs. While other major peaks belongs to MPA, the broad peak above $3000 \mathrm{~cm}^{-1}$ is assigned to $-\mathrm{OH}$ stretching of $-\mathrm{COOH}$ group and small peak at $2933 \mathrm{~cm}^{-1}$ is due to vibration of $\mathrm{CH} 2$. Peaks at $1275 \mathrm{~cm}^{-1}$ belong to $\mathrm{C}-\mathrm{O}$ bond of carboxylic acids and peaks at 1400 and $1564 \mathrm{~cm}^{-1}$ are of symmetric and asymmetric vibration of carboxylic group (-COO-) respectively. All these peaks are most distinctive bands confirming existence of carboxylic groups on surface of CdTe QDs and hence confirms that CdTe is capped by MPA, similar spectra is seen in MPA capped CdTe QDs synthesized in argon environments [6]. The presence of carboxyl groups on CdTe QDs ensures good water solubility with better fluorescence stability.

\subsection{Optical properties}

In semiconductor QDs, the size of the QDs plays a major role in optical properties, which can be understood by absorption and PL spectra. Furthermore transmission and absorption data is used to determine optical band gap of QDs and thereby useful to calculate the size of QDs. The characteristic absorption spectra of synthesized CdTe QDs aliquots collected at various reflux time are show in the Fig. 6. The absorbance spectra show a well resolved absorption peak indicating first electronic transition (1s-1s) of CdTe QDs which, red shift from $425 \mathrm{~nm}$ to $660 \mathrm{~nm}$ with increase in time, revealing the QDs growth. This profound CdTe QDs absorption peaks shift to higher energy compared to bulk CdTe material absorption peak due to Quantum confinement effect [2], which further depends on size of nanocrystals. The size of nanocrystals is influenced by reaction parameters like temperature, reflux time, $\mathrm{pH}$, capping agent and concentration of source. Monitoring these variables, the complete size ranged QDs are synthesized. Absorption peak further illustrates speed of reaction and the size distribution of QDs. The rapid shift in absorption peak wavelength at the beginning of reaction, followed by retarded shift, depicts fast growth of QDs due to nucleation and then sluggish growth due to OR process. The peak intensity and FWHM depends on the size distribution of QDs. In mono sized QDs, the peaks are well resolved and narrow compared to broad size distributed QDs. This is due to the reason that the QDs absorb same frequency as of its band gap which in turn depends on size of QDs. This can be seen at well resolved peak at $425 \mathrm{~nm}$ and the decrease as the reaction time elapse. Showing that there is narrow size distribution at first and then slight widening in size distribution as reaction proceeds.

\subsection{Photoluminescence spectra}

Photoluminescence spectra of the MPA stabilized CdTe QDs (Fig. 7) of different sizes, exhibits high luminescence with spectral range covering from green to dark red color. The PL spectra depict the higher energy electronic transition of QDs. Table 2 shows variation of photoluminescence peak wavelength and corresponding full width at half maximum (FWHM).The variation in PL intensity of different size QDs is mainly due to surface defect on the nanocrystal and size distribution of QDs. Initially surface defects in the QDs are high, due to start of fast nucleation process with high $\mathrm{Cd}$ and $\mathrm{Te}$ monomer concentration, this results in lower PL intensity (PL peak at $519 \mathrm{~nm}$ and $550 \mathrm{~nm}$ wavelength) with FWHM $\sim 65.5 \mathrm{~nm}$. PL intensity improves as reflux time increases from improved crystallization and reduced surface defect of the nanocrystal due to annealing effect (PL peaks at $584 \mathrm{~nm}$ and $638 \mathrm{~nm}$ wavelength). In addition the FWHM is least $\sim 60 \mathrm{~nm}$ showing the phase where nucleation and OR phase are in equilibrium. Further as reaction advances intensity of emission spectra diminish due to boarding in QDs size distribution and reality small surface to volume ratio (PL peak at $658 \mathrm{~nm}$ and $677 \mathrm{~nm}$ wavelength) with FWHM $66 \mathrm{~nm}$ and $85 \mathrm{~nm}$ respectively. This large increases in FWHM explicate that the OR mechanism as over took the nucleation process and growth of QDs in this stage is mainly from OR process. All though there is variation in PL intensity and FWHM; the synthesized CdTe QDs are thoroughly a good candidate for photoluminescence application mainly for biomedical labeling.

\subsection{Optical band gap and size calculation}

Optical band gap of the CdTe QDs is one of the vital parameter which determines the electrical conductivity of the material and there by its application for energy harvesting. The CdTe QDs band gap was determined by Tauc plot (Fig. 8) using equation [17].

$\alpha h v=A\left(h v-E_{g}\right)^{m}$ 


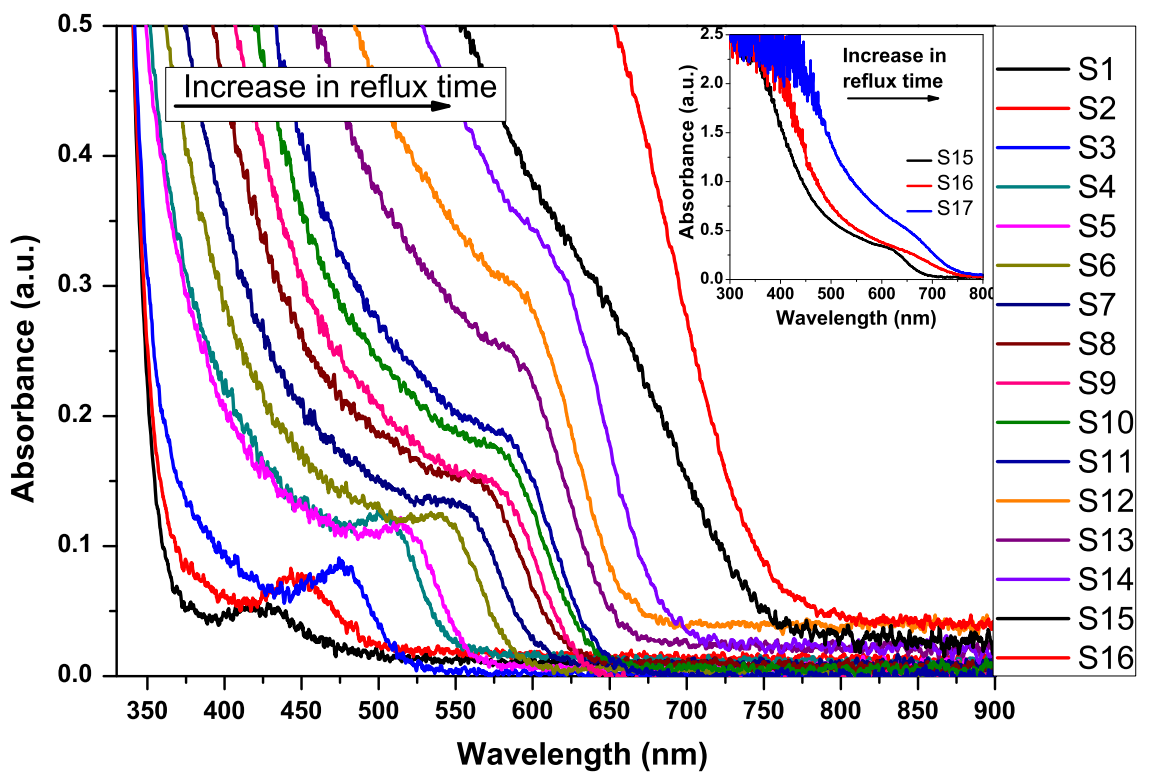

Fig. 6. Absorbance spectra of CdTe QDs. Inset shows maximum absorbance peak of CdTe QDs.

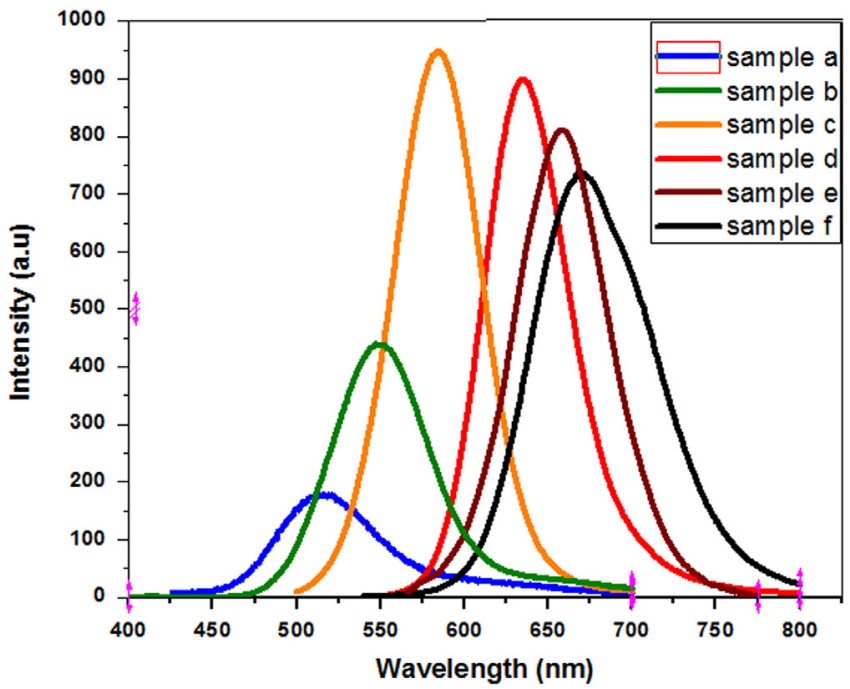

Fig. 7. Photoluminescence spectra of CdTe QDs.

Table 2

Tabulation of PL peak wavelengths, QY and FWHM of QDs sample a, b, c, d, e and $\mathrm{f}$ collected at different reflux time of $10,20,40,70,100$ and 150 minutes.

\begin{tabular}{lllll}
\hline SI. No. & Sample code & PL peak $\lambda($ in nm) & FWHM $(\mathrm{nm})$ & QY(in \%) \\
\hline 1 & Sample a & 519.446 & 65.62 & 42 \\
2 & Sample b & 550.384 & 66.04 & 48 \\
3 & Sample c & 584 & 60.89 & 59 \\
4 & Sample d & 637.55 & 59.45 & 52 \\
5 & Sample e & 658 & 66.39 & 51 \\
6 & Sample f & 677 & 84.51 & 38 \\
\hline
\end{tabular}

where $\alpha$ is absorption coefficient, $A$ is optical constant, $E_{g}$ is optical band gap, $h$ is Planck's constant, $v$ is Frequency of the incident light and $m$ show the type of transition, here $m=2$ as CdTe is direct band transition material. The optical band gap of the synthesized CdTe QDs ranges from $3.63 \mathrm{eV}$ to $1.96 \mathrm{eV}$ for first sample to final sample collected, depicting the clear red shift in band gap energy due to QDs size growth. It further reveals that, as size of QDs increases the quantum confinement energy is decreases and band gap is moving towards bulk CdTe band gap. These large band gap ranged materials are the best suitable resources for multi layered stacked solar cells and space grade solar cell to achieve high efficiency, by enhanced trapping of photons in wide spectral range. Beside its application in traditional solar cells, filters, detectors etc., band gap size of the CdTe QDs is obtained using Brus equation [1].

$\mathrm{E}_{\mathrm{g}}^{\mathrm{QD}}=\mathrm{E}_{\mathrm{g}}^{\mathrm{Bulk}}+\frac{\mathrm{h}^{2}}{8 \mathrm{r}^{2}}\left[\frac{1}{\mathrm{~m}_{\mathrm{e}}^{*}}+\frac{1}{\mathrm{~m}_{\mathrm{h}}^{*}}\right]-\frac{1.786 e^{2}}{4 \pi \epsilon_{\mathrm{o}} \in_{\mathrm{r}} \mathrm{r}}$

where: $\mathrm{E}_{\mathrm{g}}^{\mathrm{QD}} \mathrm{QDs}$ band gap $(\mathrm{eV}), \mathrm{E}_{\mathrm{g}}^{\text {Bulk }}$ bulk semiconductor band gap $(\mathrm{eV}), r$ is Radius of QDs $(\mathrm{nm}), \mathrm{m}_{\mathrm{e}}^{*}, \mathrm{~m}_{\mathrm{h}}^{*}$ is Effective mass of electron and hole respectively, $\in_{\mathrm{o}}, \in_{\mathrm{r}}$ absolute and relative permittivity respectively, $e$ charge of electron, $h$ planks constant for CdTe $\mathrm{E}_{\mathrm{g}}^{\text {Bulk(CdTe) }}=1.475 \mathrm{eV}, \mathrm{C}_{\mathrm{r}}=7.1, \mathrm{~m}_{\mathrm{e}}^{*}=0.11 \mathrm{mo}, \mathrm{m}_{\mathrm{h}}^{*}=0.35 \mathrm{mo}$, where mo is absolute mass of electron. 1st term is characteristic band gap energy of bulk materials, 2nd term gives the quantum confinement energy which increases QDs band gap energy to higher energy level compared to $\mathrm{E}_{\mathrm{g}}^{\text {Bulk }}$ directly proportional to $\mathrm{r}^{-2}$, 3rd term stand for Columbic interaction energy of exciton which reduces QDs energy level which is proportional to $\mathrm{r}^{-1}$. QDs size $(2 r)$ vs. band gap is plotted (Fig. 9). The smallest size is $2.76 \mathrm{~nm}$ and largest size of QDs is below $6 \mathrm{~nm}$. It's well known that the size calculated from Brus equation for small crystal is comparatively more than its actual size. However the largest QDs size attained is well below Bohr exciton radius $\left(a_{\mathrm{ex}}\right)$ of CdTe $\left(a_{\mathrm{ex}}=\sim 7.3 \mathrm{~nm}\right)$ [18]. Showing synthesized CdTe QDs are well under strong quantum confinement regime $\left(\mathrm{R} \ll a_{\mathrm{ex}}\right)$ and complete size ranged QDs achieved, gives rise to all the possible applications of QDs using its unique properties.

\section{Conclusion}

A good luminescent CdTe QDs with wide tunable band gap was synthesized successfully using a simple single injection hydrothermal (SIH) method completely in ambient air. The growth of QDs starts with fast nucleation followed by slow OR process. The synthesized CdTe QDs are of cubic structure, orientated in 111 plane capped with MPA, without oxidation of Te. LO peak shift in Raman spectra and strong absorption peaks with profound blue shifts compared to bulk CdTe, is attributed to Quantum confinement effect. in the synthesized CdTe QDs. High luminescence QDs with band gap varying between $3.63 \mathrm{eV}$ to $1.96 \mathrm{eV}$ and size of QDs below $6 \mathrm{~nm}$, showing synthesized CdTe QDs are in strong quantum 


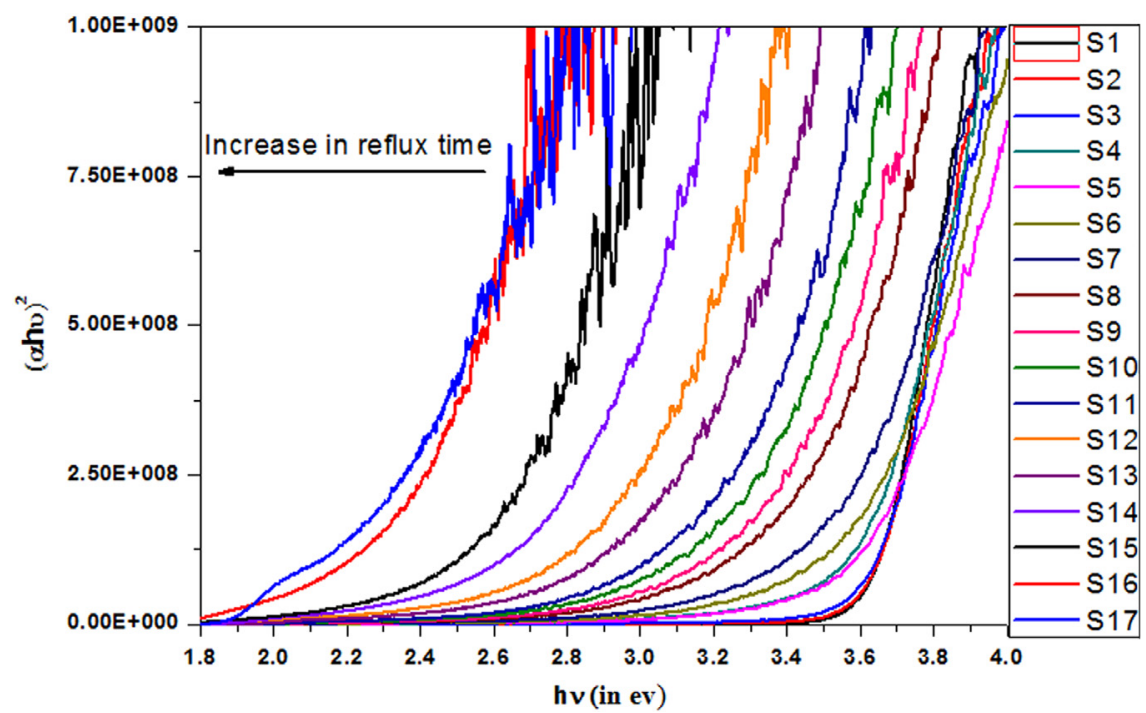

Fig. 8. Tauc plot of CdTe QDs.

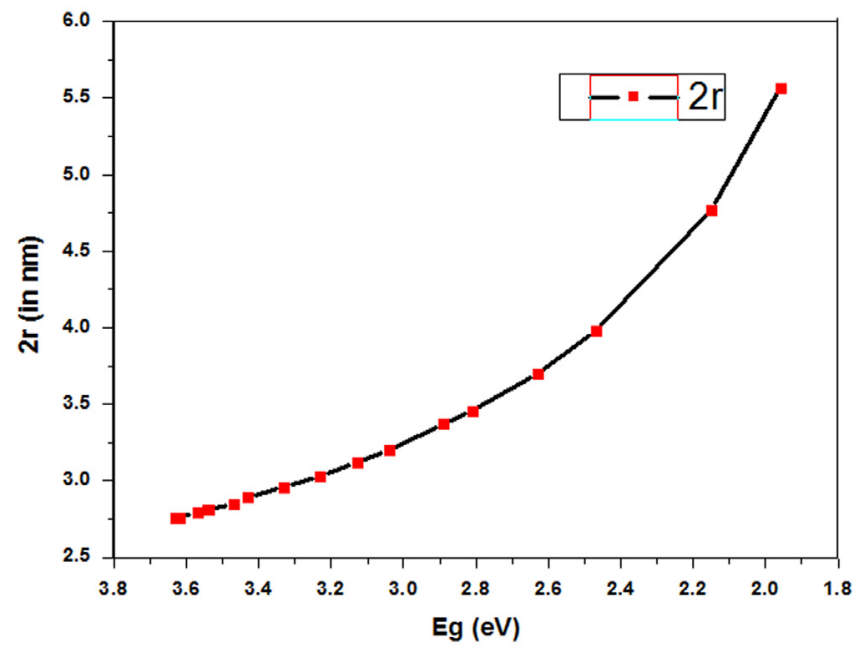

Fig. 9. Plot of calculated CdTe QDs size with respect to the band gap of the QDs.

confinement regime. Thus synthesized CdTe QDs can be used in Photovoltaic application and in biological application. The developed protocol is simple, cost effective and up scalable to industrial production.

\section{Acknowledgement}

The authors are thankful to research colleges of thin film solar cell for their support and Ms. Smitha Nair (CeNSE) and INUP, CeNSE where major portion of characterization was performed, funded by Department of Electronics and Information Technology (DeitY), Govt. of India and located at Indian Institute of Science, Bangalore.

\section{References}

[1] L.E. Brus, J. Chem. Phys. 80 (1984) 4403, http://dx.doi.org/10.1063/1.447218.

[2] A.J. Nozik, F. Williams, M.T. Nenadovic, T. Rajh, O.I. Micic, J. Phys. Chem. 89 (1985) 397, http://dx.doi.org/10.1021/j100249a004.

[3] A.J. Nozik, Advanced concepts for photovoltaic cells, in: Proceedings of the National Center for Photovoltaics and Solar Program Review Meeting, 2003.

[4] W. Shockley, H.J. Queisser, J. Appl. Phys. 32 (1961) 510, http://dx.doi.org/ $10.1063 / 1.1736034$.

[5] Y.F. Liu, J.S. Yu, J. Colloid Interface Sci. 333 (2009) 690, http://dx.doi.org/ 10.1016/j.jcis.2009.01.008.

[6] F.O. Silva, M.S. Carvalho, R. Mendonca, W.A. Macedo, K. Balzuweit, P. Reiss, et al., Nanoscale Res. Lett. 7 (2012) 536, http://dx.doi.org/10.1186/ 1556-276X-7-536.

[7] P.V. Kamat, J. Phys. Chem. C 112 (2008) 18737, http://dx.doi.org/10.1021/ jp806791s.

[8] C.B. Murray, D. Norris, M.G. Bawendi, J. Am. Chem. Soc. 115 (1993) 8706, http: //dx.doi.org/10.1021/ja00072a025.

[9] C. Ge, M. Xu, J. Liu, J. Lei, H. Ju, Chem. Commun. (2008) 450, http://dx.doi.org/ 10.1039/b714990e.

[10] R.T.R. Ribeiro, J.J.M.M. Dias, G. a G. Pereira, D.V. Freitas, P.E. Cabral Filho, R a Raele, et al., Green Chem. 15 (2013) 1061, http://dx.doi.org/10.1039/ c3gc36990k.

[11] D. Zhou, M. Lin, Z. Chen, H. Sun, H. Zhang, H. Sun, et al., Chem. Mater. 23 (2011) 4857, http://dx.doi.org/10.1021/cm202368w.

[12] Z. Sheng, H. Han, X. Hu, C. Chi, Dalton Trans. 39 (2010) 7017, http://dx.doi.org/ 10.1039/c002618b.

[13] N. Gaponik, D.V. Talapin, A.L. Rogach, K. Hoppe, E.V. Shevchenko, A. Kornowski, et al., J. Phys. Chem. B 106 (2002) 7177, http://dx.doi.org/ $10.1021 / \mathrm{jp} 025541 \mathrm{k}$.

[14] A.L. Rogach, T. Franzl, T.A. Klar, J. Feldmann, N. Gaponik, V. Lesnyak, et al., J. Phys. Chem. C 111 (2007) 14628, http://dx.doi.org/10.1021/jp072463y.

[15] W.L. Bragg, Proc. R. Soc. A Math. Phys. Eng. Sci. 89 (1913) 248, http://dx.doi. org/10.1098/rspa.1913.0083.

[16] A.K. Arora, M. Rajalakshmi, T.R. Ravindran, Encycl. Nanosci. Nanotechnol. 8 (2004) 499.

[17] J. Tauc, R. Grigorovici, A. Vancu, Phys. Status Solidi 15 (1966) 627, http://dx.doi, org/10.1016/0025-5408(68)90023-8.

[18] V. Esch, B. Fluegel, G. Khitrova, H. Gibbs, K. Kang, S. Koch, et al., Phys. Rev. B 42 (1990) 7450, http://dx.doi.org/10.1103/PhysRevB.42.7450. 